\title{
A Cross-Stack QoS Routing Approach For Underwater Acoustic Sensor Networks
}

\author{
Lloyd E. Emokpae, Zhiqiang Liu, Geoffrey F. Edelmann \\ Acoustic Signal Processing and Systems Branch \\ Naval Research Laboratory - Code 7160 \\ Washington, DC 20375 \\ lloyd.emokpae@nrl.navy.mil
}

\begin{abstract}
In this paper, we utilize a novel cross-stack design that factors in the dynamics of the underwater channel to optimize the single-hop performance amongst multiple node pairs. This will result in a set of links that meet or exceed QoS requirements, which is further leveraged for network discovery and energyefficient routing with minimum end-to-end packet delay. Thus, our proposed routing approach will provide means to guarantee application-specific QoS while also maximizing the network lifetime. Simulation experiments were conducted to validate the approach in a shallow water multipath environment.
\end{abstract}

Keywords- Underwater acoustic sensor networks, routing, energy-aware protocol.

\section{INTRODUCTION}

Underwater acoustic sensor networks (UW-ASNs) have many applications, such as environmental state monitoring, oceanic profile measurements, tactical surveillance and navigation [1]. Most of these applications require both fixed and mobile nodes in an UW-ASN to collaborate in conducting critical missions, such as patrol or search-and-rescue [2]. Moreover, unmanned underwater vehicles (UUVs) could serve as mobile command nodes or relay stations for mission critical data. These UUVs will need to cooperate in an ad-hoc fashion to be able to establish and sustain communication links, while meeting a desired level of quality of service (QoS) [1][2] and maximizing the lifetime of the UW-ASN [3]. Moreover, communications between nodes rely on acoustic links since radio signals are quickly absorbed in the underwater medium.

Although many protocols have been proposed for terrestrial wireless sensor networks (TW-SNs), they are not suitable for

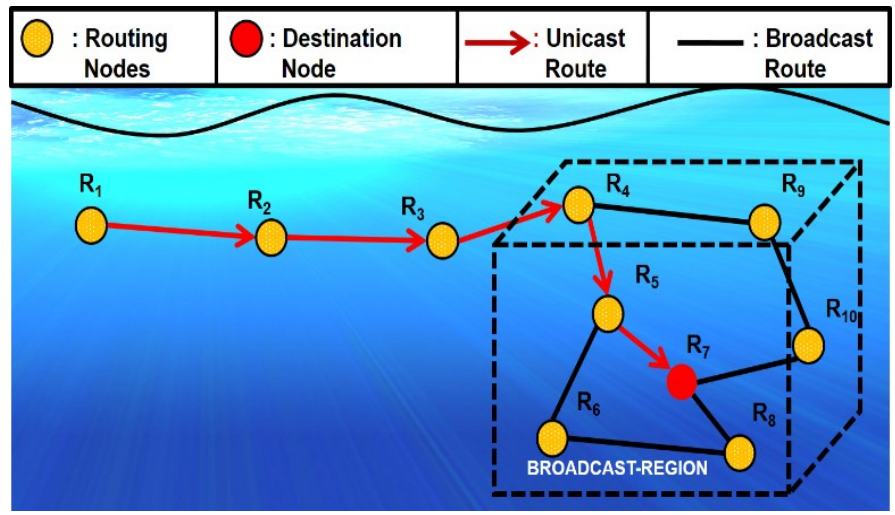

Figure 1: Illustration of the different kinds of routing options in an underwater scenario. Unicast is the most preferred option since it is more energy efficient.

\author{
Mohamed Younis \\ Dept. of Computer Science and Electrical Engineering \\ University of Maryland Baltimore County \\ Baltimore, MD 21250 \\ younis@cs.umbc.edu
}

UW-ASNs due to the limited bandwidth, long propagation delays, time-varying multipath and environmental noise. Routing algorithms will have to factor in the impacts of the underwater channel, i.e. path loss and bandwidth, on the QoS used for routing. Traditional UW-ASN networks rely on the isolated network stack structure, i.e., physical-layer, data-link layer, and network layer. The isolation between the different layers limits the prospect of optimizing the network for QoS, e.g., network throughput. In addition, when the network operates for a long duration, the energy consumption of the nodes has to be minimized. It is generally known that sensor nodes have limited onboard energy supply, making it critically important to form an energy-efficient routing topology for UWASNs.

The 3-dimentional nature of the underwater environment makes geographical routing a very popular option since nodes can leverage the known position information in the data path selection process. Nodes can exploit the relative or global position information of its neighbors to determine the best path for a packet to take based on a specific performance metric, e.g., to minimize the overall communication energy. Geographical routing can be unicast, broadcast, multicast, or anycast. Unicast is the most popular routing service in the realm of sensor networks [3][9], since only the relay node is required to forward the packet to the next node closest to the destination, as illustrated in Figure 1. To address the shortcomings of existing solutions, this paper presents a novel QoS-based routing algorithm for underwater acoustic sensor networks that is also efficient in terms of energy consumption and packet delivery latency. We utilize a novel cross-stack design that factors in the dynamics of the underwater channel to provide a set of links that meet throughput requirement, e.g., data rate needed to achieve search-and-rescue missions. We then propose a wireless acoustic line link (WALL) routing technique that exploits the identified set of links for unicast routing. WALL is validated through extensive simulation experiments and is shown to outperform competing protocols in the literature.

The remainder of the paper is organized as follows. In section II, we discuss the related work. Section III goes over our crossstack design, section IV will describe the network layer. The approach is evaluated in section V and section VI concludes the paper. 


\section{RELATED WORK}

The state-of-the-art in routing protocols for UW-ASNs can be broken down into three different categories: energy-based routing [4][5], geographic information-based routing [6][7], and hybrid routing algorithms [8][9].

Energy-based routing: In [4], the authors proposed an energy optimized path unware layered routing protocol (E-PULRP), which assumes that the sink node is stationary and is located at the center of the network. With E-PULRP, nodes that are closer to the sink and furthest away from the source node are more likely to be selected as the next-hop node. Since E-PULRP doesn't assume localization or synchronization, it relies on probe signals to be propagated for each layer to estimate the transmission ranges. This makes its implementation challenging in mobile ad-hoc scenarios. A Q-learning energyefficient and lifetime aware routing (QELAR) technique was proposed in [5], whereby nodes in the network are responsible for learning the network environment, i.e. network topology and residual energy of nodes, for path selection. The sensor node that has a larger residual energy than its surrounding neighbors, is selected as the packet forwarder. The drawback to QELAR is that it requires each node to keep track of the status of its neighbors with extra communication hand-shakes.

Geographic-based routing: A hop-by-hop dynamic addressing based (H2-DAB) routing protocol was introduced in [6] that routes data based on the location information of the sensor nodes. Unique to H2-DAB is the ability to keep the routing overhead minimum since it does not require the $3 \mathrm{D}$ coordinates, and only relies on depth information. In addition to requiring buoyancy control, H2-DAB needs to ensure that the route tables are updated in a timely manner, which directly affects the amount of information that can be transmitted. We recently proposed a geographical optimized reflection-enabled routing algorithm (GORRILA) [7], which factors in non-line-of-sight (NLOS) links in the routing process to sustain a desired QoS, i.e. network throughput. One drawback with GORRILA is that it does not consider the energy of the nodes during the routing process, especially since certain nodes along the optimized path will be heavily involved in data relaying.

Hybrid-based routing: Recently, hybrid algorithms have been proposed that aim to address the energy problem while also meeting desired network throughput QoS requirement. In [8], the authors proposed a link-state adaptive feedback routing (LAFR) algorithm that takes into account the impact of the beam width and direction of the node in the route selection. LAFR factors in the asymmetry of the underwater acoustic links due to directionality of the nodes, i.e. a node " $\mathrm{A}$ " might be within the communication range of node " $\mathrm{B}$ " but the inverse is not true. The drawback with LAFR is that the mobility of the sensor nodes is not considered. The authors in [9], proposed an energy-efficient grid routing based on 3D cubes (EGRCs) technique, which considers the unique properties of the underwater medium, i.e. 3D topology, long propagation delay, node mobility and density. EGRC makes use of residual energy, 3D locations and the end-to-end delay to determine the selection of best next-hop node. The drawback to EGRC is that it requires cluster-heads at be at pre-defined locations, which is not suitable for mobile UW-ASN routing.

\section{CROSS-STACK DeSIGN}

Recall that traditional UW-ASN networks rely on an isolated network stack structure, i.e., physical-layer, data-link layer, and network layer. This isolation limits us from an optimizing the network for a specific QoS, e.g., network throughput. To overcome this limitation, we promote a novel cross-stack design, whereby the physical layer is crossed with the data-link layer with the network layer on top. Our crossstack will enable the identification of a set of links that meet or exceed desired throughput requirement. The network layer will then exploit this information to establish an energy-efficient routing. The next subsections will go over the different portions of our cross-stack model, highlight some assumptions, and define the different QoS metrics, i.e., throughput and energy, used in our routing process.

\section{A. Single-Hop Throughput and Energy Model}

Our cross-stack model aims to jointly designing the physical and data-link layers for optimum single-hop network throughput. Whereby any excess throughput, e.g. exceeding our QoS requirements, will be used to improve energy during routing. Our design assumes that we are using a slotted carriersense multiple-access with collision avoidance (CSMA/CA) protocol. Thus, we can define our saturation throughput according to [10] as:

$$
\begin{aligned}
& T H=\frac{\text { Average payload transmitted during one slot }}{\text { Average slot duration }} \\
& T H=\frac{p_{t r} p_{s} E[P]}{\left(1-p_{t r}\right) \sigma+p_{t r}\left(1-p_{s}\right) T_{c}+p_{t r} p_{s} T_{s}}
\end{aligned}
$$

where $E[P], \mathrm{T}_{\mathrm{c}}, \mathrm{T}_{\mathrm{s}}, \sigma, p_{t r}$ and $p_{s}$ corresponding to the average payload size, the average collision duration, the average successful transmission duration, the duration of an empty time slot, the probability that at least one node is actively transmitting and the probability of a successful transmission, respectively. These probabilities have been fully analyzed in [10], which show that the throughput is proportional to $p_{s}$. This probability is a function of the probability of signal outage $p_{\text {outage }}$ and signal capture $p_{\text {capture }}$, which can be estimated from previous acoustic communication messages, i.e. via maximum likelihood estimation method. This can be further simplified to a least squares solution if the probability density function of $p_{\text {outage }}$ and $p_{\text {capture }}$ follow a uniform distribution. Each node will estimate this throughput parameter from their nearest single-hop neighbor by measuring the quality of the acoustic channel for $p_{\text {outage }}$ estimation while keeping track of the number of single-hop contenders for $p_{\text {capture }}$ estimation. In addition, any excess throughput will be used to improve the energy consumption when performing unicast route.

We adopt an energy consumption model that incorporates both the line-of-sight (LOS) and the multipath transmission losses. The energy consumption of nodes in the UW-ASN is mainly due to communication and data processing. Thus, we 
can define the energy (in joules or $\mathrm{J}$ ) required for transmission as the change in power $\Delta P$ time's change in time $\Delta t$. This is effectively the sum of the transmission electrical energy $\mathrm{E}_{\mathrm{ELEC}-\mathrm{TX}}$ and the communication energy $\mathrm{E}_{\mathrm{COMM}}$, i.e.

$$
\begin{aligned}
& \mathrm{E}_{\mathrm{TX}}=\Delta P \cdot \Delta t=\mathrm{E}_{\mathrm{ELEC}-\mathrm{TX}}+\mathrm{E}_{\mathrm{COMM}} \\
& \mathrm{E}_{\mathrm{TX}}=\mathrm{E}_{\mathrm{ELEC}-\mathrm{TX}}+l \cdot d^{2} \alpha(f)^{d}
\end{aligned}
$$

where $l$ is the number of packet bits, $d$ is the LOS distance in meters and $\alpha(f)$ stands for Thorps absorption coefficients, given by [9]:

$$
\begin{gathered}
\log \alpha(f)=0.011 \frac{f^{2}}{1+f^{2}}+4.4 \frac{f^{2}}{4100+f^{2}}+2.75 \times \\
10^{-5} f^{2}+0.0003
\end{gathered}
$$

Similarly, the energy consumption for the receiver node is:

$$
\mathrm{E}_{\mathrm{RX}}=\mathrm{E}_{\mathrm{ELEC}-\mathrm{RX}}
$$

where $\mathrm{E}_{\mathrm{ELEC}-\mathrm{RX}}$ denotes the reception electrical energy. To put (3) and (5) in perspective, most commercial underwater acoustic amplifiers for mid-range communications $1 \mathrm{kHz} \leq f \leq$ $50 \mathrm{kHz}$ require $80 \mathrm{~W}$ of power at over $90 \%$ efficiency with an 8 ohm load [12]. Hence, for a packet duration of 1s, we will require $80 \mathrm{~J}$ of electrical energy during transmission. The electrical energy required during reception mainly consists of low-noise-pre-amplifiers and passive components, which can be shown to consume less than $1 \mathrm{~J}$ of energy for most modern systems.

\section{NETWORK LAYER}

Each node in the network will exploit the cross-stack model defined in section III-c, which will provide the network layer with a means to assess the throughput for a communication link between a pair of nodes in order to identify a set of nodes that meet or exceed the throughput requirement, i.e., $T H\left(R_{i}\right) \geq \lambda$, for $R_{i}$ and $\lambda$ being the $\mathrm{i}$-th neighbor and QoS metric, specifically throughput, respectively. Using such a set of links, the goal of the network layer is to determine the best path for the packet that satisfies the QoS metric. We adopt a hybridbased routing methodology, whereby a node uses the geographical information from its QoS-qualified neighbors to determine energy-efficient paths. For our purposes, we will assume that each node will perform a suitable localization method similar to [11] in order to determine the relative positions of their neighbor's up to k-hops away. We will exploit the known position information in our network discovery mechanism. Due to space constraints, the reader is referred to [11] on details on closed-form localization techniques that can be applied for topology discovery purposes. The next section will describe the unicast routing method used in WALL.

\section{A. Wireless Acoustic Line Link: Unicast Routing}

We will use the constructed planar graph from the relative node positions during discovery to perform unicast routes that will guarantee our throughput QoS while (a) taking the quickest path to the destination or (b) taking routes that maximizes the network lifetime. We define our graph $G$ with edge set $E$, such that the edge weights range from $[0, \infty]$ represents the cost of the link. Thus, the goal is to select the next-hop routing node in the subset of routing nodes $R$, i.e. $R_{i} \subset R$, that meet the minimum throughput QoS and has minimum edge weight or link-cost. If this is the first packet, the source node will reset the edge weights of the graph to be equal to 1 , the new packet will then be generated based on the agreed upon packet structure.

A breadth first search (BFS) will then be conducted on the graph by biasing the search towards the destination node, i.e. applying $\beta$ weights. This will result in the search returning the least-cost path. If we always want to use the quickest path, we simply set $\beta=1$, otherwise we set $\beta \leq-1$ such that the next time around the BFS will result in a path that is different from the previous shortest path, thereby distributing the energy consumption across other nodes in the network. In the next section we will evaluate WALL through simulation and compare it against the state-of-the-art techniques discussed in the related work section.

\section{Perfromance Evaluation}

In this section we validate the performance of the WALL routing scheme to GORRILA [7], which is a geographical routing approach, and to EGRC [9], which is a hybrid approach. The simulations were conducted in Matlab with the cross-layer parameters defined in Table 1. The underwater environment represents a cube with dimensions of $200 \mathrm{~m} \times 200 \mathrm{~m} \times 200 \mathrm{~m}$, where the water surface is modeled as a non-flat surface with root-mean-squared roughness of approximately 0.5 . The water bottom is also modeled as a rough surface with a silt-like material $\left(p_{b} / p_{w}=1.7\right)$. We randomly placed $\mathrm{N}$ nodes and studied the energy, throughput and hop-count performance as we vary the number of nodes in the network. Our energy metric of success is the minimum residual energy (MRE) of a node in the network, which effectively gauges the network lifetime. We can define this metric as:

$$
M R E=\min \left(E\left(R_{1}\right), E\left(R_{2}\right), \ldots E\left(R_{N}\right)\right)
$$

where, $E\left(R_{i}\right)$ is the residual energy of the node $R_{i}$. We assume that each node has an initial energy based on the battery capacity. We then set $\beta$ to -1 to bias the route for energyefficiency. Finally, we report the results where we observe that within a $90 \%$ confidence level, the energy (in J), normalized throughput and hop-count all stay within $10 \%$ of the sample mean

\section{A. Performance Comparisons}

Figure 2-a shows a plot of the energy performance as we vary the number of nodes $N$. We see that WALL outperforms

\begin{tabular}{|c|c|c|c|}
\hline$k_{L O S}$ & $c_{w}(\mathrm{~m} / \mathrm{s})$ & $c_{b}(\mathrm{~m} / \mathrm{s})$ & $\mathrm{z}_{\mathrm{T}}$ \\
\hline $150 \mathrm{~m}$ & 1500 & 1800 & $-1 \mathrm{~dB}$ \\
\hline$f_{c}(\mathrm{kHz})$ & $p_{w}\left(\mathrm{~kg} / \mathrm{m}^{3}\right)$ & $p_{b}\left(\mathrm{~kg} / \mathrm{m}^{3}\right)$ & $\mathrm{N}_{\mathrm{T}}$ \\
\hline 1 & 1000 & 1700 & $-90 \mathrm{~dB}$ \\
\hline$u(\mathrm{bps})$ & $\mathrm{P}_{\mathrm{TX}}$ & $\mathrm{E}_{\mathrm{INIT}}{ }^{*}$ & $\mathrm{E}_{\mathrm{ELEC}}$ \\
\hline 1000 & $20 \mathrm{~dB}$ & $343 \mathrm{~kJ}$ & $120 \mathrm{~J}$ \\
\hline $\mathrm{z}_{\mathrm{T}}:$ Received signal threshold \\
$c_{w} / p_{w}:$ Sound-speed/Density in the water surface layer \\
$c_{b} / p_{b}:$ Sound-speed/Density in the water bottom layer (silt-like bottom) \\
$\mathrm{P}_{\mathrm{TX}}:$ Transmission power \\
$\mathrm{N}_{\mathrm{T}}:$ Noise power \\
$f_{c}:$ Acoustic carrier frequency \\
${ }^{*} E_{I N I T}$ : Based on a 95Wh Ocean-Server ${ }^{\mathrm{TM}}$ battery capacity
\end{tabular}
Table 1: Cross-layer simulation parameters 


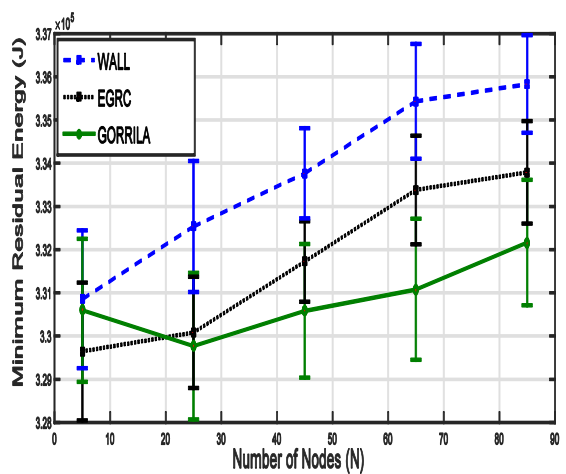

Figure 2: (a) Energy performance comparison. Larger network densities will allow for more relay options thereby increasing the MRE.

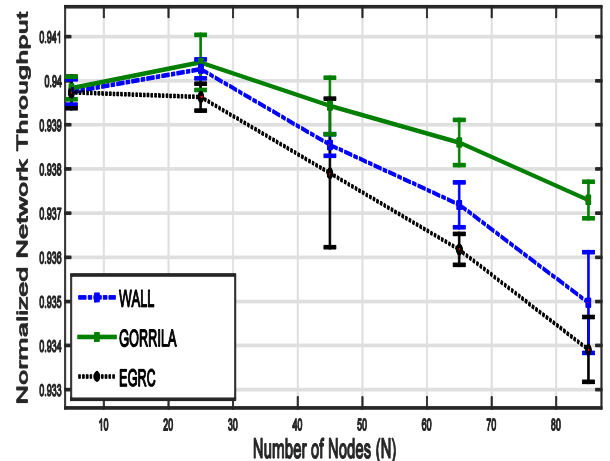

(b) Normalized throughput performance, showing that WALL outperforms competing hybrid schemes but sacrifices excess throughput for improved MRE.

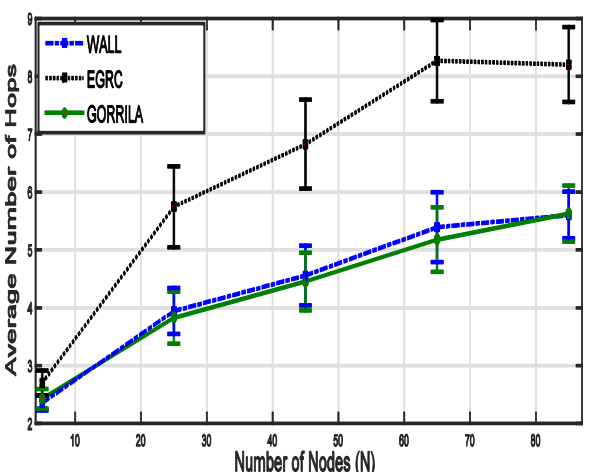

(c) The hop-count performance results show that WALL gravitates towards the shortest path with less hops needed to route packet. both ECGR and GORRILA in terms of MRE after transmitting 25 packets across the network. For lower network densities, i.e. small $N$, certain nodes will take on a majority of the burden as packet relays, which will result quicker depletion of their residual energy. However, as we increase $N$, the algorithm will result in more paths thereby distributing the load of the traffic across the network. The normalized throughput performance is shown in Figure 2-b, we see that in all three cases the network reaches a saturation point at about 20 nodes with diminishing returns afterwards. More importantly, we see that WALL outperforms the state-of-the-art hybrid scheme but sacrifices excess throughput for improved MRE. Lastly, we see the hopcount performance in Figure 2-c. The hop-count is proportional to the end-to-end packet delay and measures the travel time of each packet. From the figure, we see that as we increase the network density the hop-count increases, which is expected. More importantly, we note that WALL uses fewer hops (for $N$ $>5$ ) than ECGR to route the packet to the destination. Figure 3 shows the energy consumption of the network discovery, which is mainly due to the acoustic communications needed for neighbor localization. The plot was generated by varying the minimum distance between nodes. The plot shows that the energy consumption is bounded between $150 \mathrm{~J}$ and $200 \mathrm{~J}$ depending on the network discovery accuracy needed.

\section{CONCLUSION}

In this paper we have introduced a novel cross-stack design that factors in the dynamics of the underwater channel to optimize the single-hop performance amongst multiple node pairs to

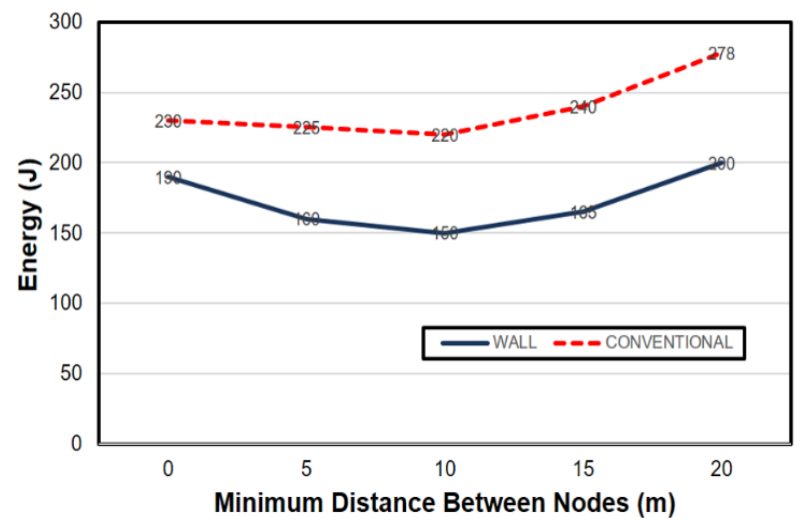

Figure 3: Graph construction energy performance over conventional means. In fixed networks, a majority of the energy consumption will be due to the meet some desired QoS performance. We have then presented a novel wireless acoustic line link (WALL) routing approach that exploits the set of links in addition to the known relative positions of the nodes to construct a graph of the network based on QoS qualified links. Multi-hop data paths are selected to maximize the total network lifetime and minimize end-to-end delay. The simulation results have confirmed the effectiveness of WALL and shown improved energy, throughput and hopcount performance over the state-of-the-art routing schemes.

\section{ACKNOWLEDGEMENTS}

This work was supported by the Office of Naval Research.

\section{REFERENCES}

[1] L. Liu, S. Zhou, and J.H. Cui, "Prospects and Problems of Wireless Communications for Underwater Sensor Networks," Wireless Communications and Mobile Computing, Special Issue on Underwater Sensor Networks, Vol. 8, No. 8, pp. 977-994, May 2008.

[2] I. F. Akyildiz, D. Pompili, and T. Melodia, "Underwater Acoustic Sensor Networks: Research Challenges," Ad Hoc Networks, 3(3), pp. 257-279, Ma. 2005.

[3] M. Zorzi, P. Casari, N. Baldo, and A. Harris III, "Energy-Efficient Routing Schemes for Underwater Acoustic Networks," IEEE Journal on Selected Areas in Comm., 26(9), pp. 1754-1766, Dec 2008

[4] S. Gopi et al. "E-PULRP: Energy Optimized Path Unaware Layered Routing Protocol for Underwater Sensor Networks," IEEE Trans. Wireless Communications, 9(11), pp. 3391-3401. Sept 2010

[5] T. Hu and Y. Fei, "QELAR: A Machine-Learning-Based Adaptive Routing Protocol for Energy Efficient and Life-time-Extended Underwater Sensor Networks," IEEE Trans. Mobile Comp. vol. 9, No. 6, pp. 796-809. Feb 2010.

[6] M. Ayaz, A. Abdullah, I. Faye and Y. Batira, "An Efficient Dynamic Addressing Based Routing Protocol for Underwater Wireless Sensor Networks," Computer Communications, 35(4), pp. 475-486, Feb. 2012

[7] L. Emokpae and M. Younis, "Signal Reflection-enabled Geographical routing for underwater sensor networks," Proc. of International Conference on Communications (ICC'12), pp. 1-6, Jun 2012.

[8] S. Zhang, D. Li and J. Chen, "A Link-State Based Adaptive Feedback Routing for Underwater Acoustic Sensor Networks," IEEE Sensors Journal, vol. 13, no. 11, pp. 4402-4412, Nov 2013

[9] K. Wang, H. Gao, X. Xu, J. Jiang and D. Yue, “An Energy-Efficient Reliable Data Transmission Scheme for Complex Environmental Monitoring in Underwater Acoustic Sensor Networks," IEEE Sensors Journal, 16(11), pp. 4051-4062, Jun 2016.

[10] L. Emokpae and M. Younis, "Throughput Analysis for Shallow Water Communication Utilizing Directional Antennas," IEEE Trans. on Selected Areas in Communications, 30(5), pp. 1006-1018, Jun 2012

[11] L. Emokpae, S. DiBenedetto, B. Potteiger and M. Younis, "UREAL: Underwater Reflection-Enabled Acoustic-based Localization," IEEE Sensors Journal, 14(11), pp. 3915-3925, September 2014

[12] Amplifier, http://www.icepower.dk/files/solutions/icepower250adata.pdf 